\title{
EL UNIVERSO NÁVAJO Y LA EXPLOTACIÓN DE SUS RECURSOS NATURALES EN EL SIGLO ACTUAL
}

\author{
POR \\ MARÍA PILAR ALONSO-NÚÑEZ
}

Los Návajos son el grupo más grande de nativos americanos que viven en una reserva en los Estados Unidos. Según el Censo de los Estados Unidos del año 1990 hay 219.198 Návajos en todo el país. Según cifras publicadas por Wilkins, en 1987, había 192.000 «ciudadanos» Návajos en los Estados Unidos. De éstos, 149.000 viven en la reserva propiamente dicha, 22.000 viven en las tierras adyacentes, y aproximadamente 22.000 viven en un buen número de ciudades estadounidenses (1987: xv). Sin lugar a dudas, uno de los grandes problemas que amenazan al mundo návajo es la explotación de los recursos energéticos de su reserva.

Hay muchos trabajos publicados sobre las consecuencias devastadoras que esta explotación indiscriminada está teniendo sobre el medio ambiente y sobre la salud de los Návajos. Entre ellos podemos destacar los realizados por Suzzane Gordon, Black Mesa. The Angel of Death (1973); Solomon Norman y Harvey Wasserman, Killing Our Own. The Disaster of America's Experience With Atomic Radiation (1982); Ward Churchill y Winona LaDuke, «Native North America: The Political Economy of Radiactive Colonialism» $(1992,1996)$; Ward Churchill «The Earth is Our Mother: Struggles for American Indian Land and Liberation in the Contemporary United States (1992, 1996); Ward Churchill, «Genocide in Arizona? The "Navajo-Hopi Land Dispute" in Perspective»

María Pilar Alonso-Núñez. Native American Studies Departament. Universidad de California, Davis.

Estudios Geográficos

Tomo LX, n. ${ }^{\circ} 235$, abril-junio 1999 
(1993, 1996); Peter H. Eichstaedt, If You Poison Us. Uranium and Native Amricans (1994). Si bien estos trabajos son de extrema importancia para denunciar un hecho que está aconteciendo en la reserva actualmente, en este trabajo, aunque se hará mención a las consecuencias devastadoras desde el punto de vista del medio ambiente y de la salud de los Návajos, se centrará en el significado que para ellos tiene esa tierra, que está dentro de lo que ellos consideran es su Quinto Mundo o actual ${ }^{1}$. De esta forma se entenderá por qué esa explotación está llevando consigo más que la destrucción física de la tierra, está llevando consigo la destrucción de las creencias que en ella se encuentran. Como dice MacPerson (1992: 3), «Esa tierra y las creencias que van con ella riegan y fertilizan la mente del Diné (Návajo), ayudándole a mantener su identidad en el "centro" del mundo» (Fig. 1).

El significado de la tierra para el Návajo se encuentra en las historias orales que han sido transmitidas de padres a hijos desde tiempos inmemoriales. Historias que acontecieron en un espacio delimitado entre cuatro montañas sagradas, Blanca Peaks, Mount Taylor, San Francisco Peaks y Hesperus Mountain, que forman lo que el Návajo considera son los límites de su mundo actual. La tierra entre esas cuatro montañas y las historias que en ella acontecieron forman lo que podemos considerar es «el libro sagrado» del Návajo. Como dicen los Návajos, sin las historias, la tierra es una tierra más, pero con ellas, ésta toma un significado distinto y se convierte en sagrada para sus habitantes.

Un trozo de tierra es como un libro. Una persona sabia puede mirar a las rocas y a las montañas y leer las historias que son más antiguas que cualquier cosa viva que existió en la superficie de la tierra... (Evers, 1994: 1).

"El territorio entre las cuatro montañas sagradas es un regalo de los seres sobrenaturales»

De acuerdo con las creencias de los Návajos, el mundo actual es considerado el Quinto, habiendo habido cuatro anteriores que fueron destruidos porque sus ancestros no siguieron las reglas de comportamien-

${ }^{1}$ Hay Návajos que piensan que este es el Cuarto Mundo, otros hablan de 12 mundos di-
vididos en tres niveles. 


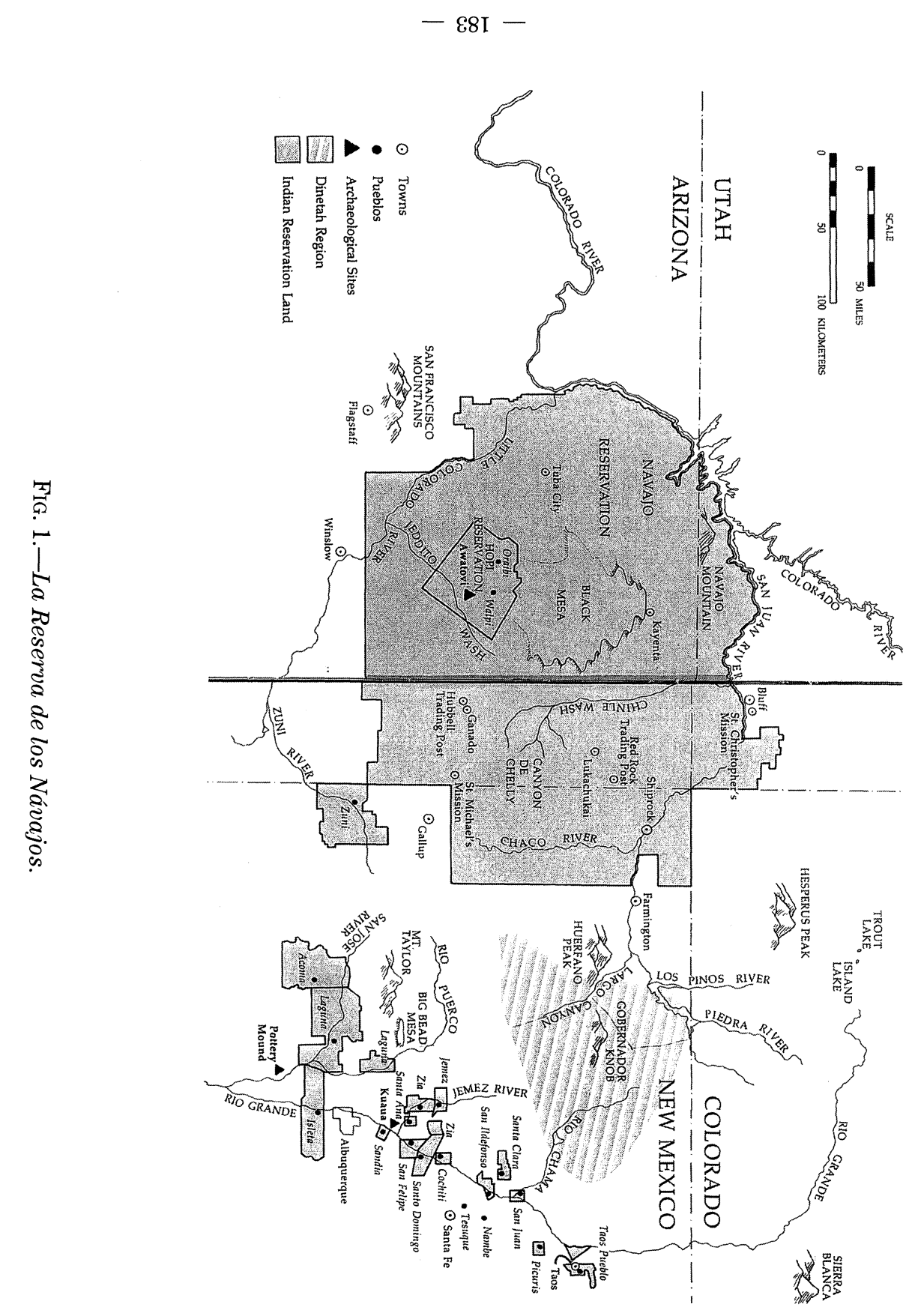

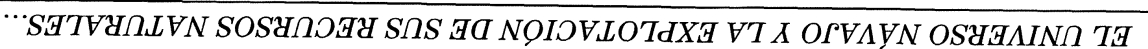


to que los creadores del mismo les habían dado. Este Quinto Mundo tiene unas fronteras físicas que son cuatro montañas, consideradas sagradas por los Návajos, en el Este Blanca Peaks, en el Sur Mount Taylor, en el Oeste San Francisco Peaks y en el Norte Hesperus Mountain.

La historia de la creación de este mundo actual se encuentra recogida en una ceremonia conocida en návajo como el nombre de Hózhóójí, en inglés como Blessingway, y que podemos traducir en español como Acto de Bendición. Esta ceremonia cuenta cómo, quién y para quién se creó el mundo actual.

Hózhóóji cuenta que después de la destrucción del Cuarto Mundo, un grupo de Diyin Dine'é, de seres sobrenaturales entre los que estaba el Primer Hombre, la Primera Mujer, el dios Llamador y dios Hablador llegaron a la superficie del mundo actual ${ }^{2}$. Allí construyeron un hogan (casa tradicional návaja) donde comenzaron a pensar en planear el mundo actual para los ancestros de los Návajo actuales ${ }^{3}$.

Según Witherspoon (1975: 71), el pensamiento para los Návajos tiene el poder de crear, transformar y regenerar, ya que este mundo fue creado a través del mismo. Frank Mitchell, en su versión de Hózhóójí dice: $344)$.

Y así, ya ves, todo lo que fue pensado se hizo realidad (Wyman, 1970:

Aronilth, un hombre médico actual dice:

Ahora, hoy todo lo que fue pensado por la Gente Sagrada existe en realidad. Por consiguiente fue bueno (Aronilth S. F.: 1-2).

Esta opinión también es compartida por uno de mis informantes quien me dijo: «La Gente Sagrada en el interior de la casa (hogan)

2 Wyman identifica el lugar de la Emergencia con Trout Lake localizado en las Montañas de San Juan en el estado de Colorado (1957: 37). Por otra parte, Watson dice que, aunque Trout Lake es considerado como un lago sagrado, no es el lago a través del cual los návajos emergieron a este mundo. La razón es que este lago no tiene una isla en el medio, como la tradición oral dice que tenía el lago de la Emergencia (1964: 18). De acuerdo con otros investigadores la localización exacta de este lugar se piensa que está cerca de Návajo Lake (Witherspoon and Peterson, 1995: 26).

${ }^{3}$ Los Návajos fueron creados por la Mujer Cambiante cuando el Quinto Mundo estaba terminado. 
creó la vida con su pensamiento» (Comunicación Personal, julio de 1995).

La versión de Slim Curly comienza con la narración de Las Formas Interiores que Habrá.

Cuando ellos (El Primer Hombre y la Primera Mujer) estaban planeando (pensando) cómo iban a ser las condiciones de vida en el futuro... Habrá formas interiores del Sol y de la Luna... Y también habrá formas interiores de La Mujer del Agua, de la Mujer de la Montaña ${ }^{4}$. Y también de los puntos cardinales del Este, Oeste, Sur y Norte, y habrá formas interiores de esos (fenómenos naturales) que los rodean (los puntos cardinales) en el sentido del sol (es decir de Este a Oeste)... (Wyman, 1970: 109-110).

Entonces vemos que lo que el Primer Hombre, la Primera Mujer y los otros (se refiere al resto a los seres sobrenaturales que vinieron con ellos) crearon con sus pensamientos fueron las formas interiores de los fenómenos naturales que iban a tener una posición prominante en la estructura y operación de este mundo (Whiterspoon, 1975: 71). De hecho, las cuatro montañas sagradas que forman los límites de lo que los Návajos consideran es su Quinto Mundo eran las mismas montañas que existían en los mundos anteriores.

A través de la ceremonia Hózhóójí tenemos suficiente información que nos dice que los fenómenos naturales son la morada de esas formas interiores, de los seres sobrenaturales.

En la mayoría de los casos la Gente Sagrada del Quinto Mundo son las formas interiores de varios fenómenos naturales y fuerzas, incluyendo los animales, y son (la Gente Sagrada) los poderes que controlan y animan la naturaleza. El ritual Návajo no está designado para controlar los elementos directamente; está designado para controlar la Gente Sagrada, que son las formas interiores y los agentes controladores de esos elementos (los fenómenos naturales) (Witherspoon 1977: 35).

Por lo tanto cuando en Hózhóóji se habla de «la creación de las formas interiores» en realidad se refiere a la creación de las formas o se-

\footnotetext{
${ }^{4}$ Cuando se habla de la Mujer del Agua y de la Mujer de la Montaña, no se refieren a cuerpos específicos de agua o de las montañas, sino a todos los cuerpos de agua y de montañas que existen en el mundo de los Návajos (Wyman, 1970: 110, Nota 89). 
res sobrenaturales que van a ocupar los distintos fenómenos naturales.

De acuerdo con la versión de Frank Mitchel (Wyman, 1970: 344), lo primero que pensaron el Primer Hombre, la Primera Mujer, y el grupo de seres sobrenaturales que les acompañaban, fue colocar a la Tierra y al Cielo en la posición en que los vemos actualmente (implicando que ya existían). Posteriormente pensaron decorar el cielo y la tierra con los distintos elementos que vemos ahora en ellos, como el sol, la luna, las constelaciones, las montañas, los ríos, la vegetación, etc., todo lo que en el futuro iba a ser útil para los ancestros de los Návajos actuales.

Cuando empezaron a pensar este mundo, el Primer Hombre sacó de su vestido un hatillo que contenía las cuatro «joyas» o «piedras» que son sagradas para los Návajos: la turquesa, concha blanca, el abalon y azabache, esculpidas en forma de mazorcas de maíz ${ }^{5}$. El Primer Hombre abrió el hatillo y pasó sus manos sobre las cuatro «joyas»o «piedras»; posteriormente se tocó el cuerpo con sus manos y las puso en su boca y volvió a tocar las «joyas» o «piedras» (Wyman, 1970: 110-113). Estas «joyas»o «piedras» se convirtieron en los seres sobrenaturales que posteriormente ocuparían el interior de los distintos fenómenos naturales y que a menudo se mencionan en Hózhóójí.

Posteriormente el Primer Hombre y su grupo, decidieron crear las montañas que iban a ser los límites del Quinto Mundo, así como el sol y la luna. Las montañas las crearon para poner a la Tierra y al Cielo en la posición firme en que los vemos actualmente (Wyman, 1970: 351).

El Primer Hombre volvió a sacar su hatillo, el cual contenía pequeños puñados de tierra de las montañas que habían existido en el Cuarto Mundo ${ }^{6}$ (Wyman, 1970: 351).

En el Este, el Primer Hombre y el grupo de seres sobrenaturales que le acompañaban, pusieron Sisnaajini Blanca Peaks ${ }^{7}$, en el Sur pusieron

${ }^{5}$ Las «joyas» o «piedras» son fuentes de vida en los actos de creación de los Návajos (Farella, 1984: 73).

${ }^{6}$ Algunos hombres médicos dicen que esas montañas existían desde el Primer Mundo, y que fue en el Quinto Mundo cuando se convirtieron en sagradas. Otros hombres médicos (Comunicación Personal, junio de 1995) dicen que estas montañas han sido siempre sagradas, desde el Primer Mundo.

7 La identificación de la montaña del Este, conocida en Návajo con el nombre de Sis Naajini ha sido objeto de una gran controversia entre los investigadores. Matthews (1994: 78, Nota 52) dice que Tsisnadzi'ni (Sis Naajinそ) es alguna montaña cerca del Pue- 
Tsoodzil, Mount Taylor, en el Oeste Dook' o'ooslíid, San Francisco Peaks y en el Norte Dibé Nitsaa, Hesperus Peak ${ }^{8}$ (Wyman, 1970: 351-352).

Estas cuatro montañas marcan los límites de lo que los Návajos consideran es su mundo actual, y coinciden con los cuatro puntos cardinales. George Blueeyes, un hombre médico, nos dice de estas montañas:

Ellas (las montañas) fueron puestas aquí para nosotros. Nosotros pensamos de ellas como nuestra casa.

Esas montañas y la tierra en medio de ellas son las únicas cosas que nos mantienen fuertes. De ellas, y por ellas nosotros prosperamos por ellas comemos plantas y buena carne.

Nosotros tenemos tierra de las Montañas Sagradas en un hatillo para rezar, al cual nosotros llamamos nídiilyééh.

Es por ese hatillo que tenemos ovejas, caballos y vacas. Por este hatillo, nosotros tenemos posesiones de mucho valor, turquesas, collares y brazaletes. Con el hatillo rezamos, con el hatillo los rezos comienzan (Evers, 1994: 2).

Una vez que colocaron las montañas como las vemos actualmente, el Primer Hombre y la Primera Mujer tuvieron una discusión con respecto a las formas interiores, es decir a los seres sobrenaturales, de las montañas:

Me pregunto como vamos a hacer para que esas montañas, que hemos creado sean útiles para los futuros Návajos. Vamos a poner algunos seres

blo de Jeméz, en el Condado de Bernardillo, en el estado de Nuevo México. Probablemente sea Pelado Peak, opinión también compartida por los padres franciscanos (Franciscan Fathers, 910: 136), Sleight (1951: 395) y por Amsden (1932: 195). Otros investigadores como Haile (1938: 66) y Watson (1964: 7) identifican esta montaña con Blanca Peak en las montañas de Sangre de Cristo, cerca de Alamosa, en el estado de Colorado, opinión también compartida con los autores (todos ellos Návajos) del libro Between Sacred Mountains, pp. 2-3. Wyman (1957: 37) identifica esta montaña con Pelado Peaks en las Montañas Jeméz de Nuevo México, argumentando que Blanca Peaks está más al norte que la montaña sagrada del Norte, Hesperus Mountain. Para ver más de esta controversia sobre la localización de la montaña del Este, ver el artículo de Sleight, «The Navajo Sacred Mountain of the East-A Controversy».

$8 \mathrm{Al}$ igual que con la montaña del Este la localización de esta montaña es dudosa. Wyman (1970: 18-19) dice que el nombre de esta montaña en Návajo es Dibén'tsaa y significa Big Sheep (cabra montesa) y parece referirse a la abundancia de esas cabras en las Montañas Rocosas. Quizás haga referencia a la cantidad de cabras montesas que estas montañas ofrecían a las antiguas tribus cazadoras (Wyman, 1970: 128, nota 114). 
que vayan a vivir al interior de ellos. La verdad es que si no hay nadie viviendo en su interior ellas (las montañas) son nada más que cosas que están por ahi sin ningún propósito (Wyman, 1970: 357).

Veamos a continuación como Hózhóóji nos cuenta cómo las formas interiores, los seres sobrenaturales, que iban a vivir en el interior de las montañas, fueron creados.

De acuerdo con la versión detallada de Frank Mitchell (Wyman, 1970: 357-358), el Primer Hombre preguntó a dios Hablador y a dios Llamador: ¿Quién de vosotros está dispuesto a estar en el interior de esas montañas? Ninguno de los dos estaba disponible por el momento ${ }^{9}$. Entonces decidieron que iban a crear «las formas interiores» de las montañas a imagen y semejanza de estos dos «dioses».

El dios Hablador comenzó a pensar cómo lo harían, y puso en el suelo una concha blanca, y una turquesa, sobre las que aplicó agua de rocío que había traído de la montaña del Este, es decir de Blanca Peak y rocío que había traído de la montaña del Sur, de Mount Taylor. El dios Hablador las santificó con sus manos y de esas «piedras» o «joyas» salieron diferentes personas que se convertirían en las formas interiores de esas montañas ${ }^{10}$. Hózhóóji nos describe estos personajes de la siguiente manera:

De la montaña del Este salió un ser como el Dios Hablador. Él se levantó con una piel de ciervo blanca sobre su hombro derecho que caía sobre su cadera derecha y con un tocado de doce plumas blancas... Entonces el Dios Hablador sopló su voz sobre este ser (Wyman, 1970: 360).

El Dios Hablador hizo lo mismo en la montaña del Sur para crear un Dios Hablador de Turquesa... El (el nuevo Dios Hablador) llevaba una falda y su tocado consistía de doce plumas amarillas. Otra vez el Dios Hablador sopló su voz sobre él (Wyman, 1970: 360).

La versión de Frank Mithchell (Wyman, 1970: 361, nota 258) no menciona los nombres de los seres sobrenaturales que fueron creados por el

${ }^{9}$ Siendo los encargados de dar la vida a los fenómenos naturales tenían que seguir trabajando más hasta que la creación de este Quinto Mundo estuviera completa.

${ }_{10}$ El agua de rocío al igual que las cuatro «joyas» 0 «piedras» sagradas, son fuentes de vida en los actos de creación de los Návajos. El rocío viene del cielo y de los puntos cardinales, de los fenómenos cardinales. El agua de rocío se conoce en Návajo con la palabra Yadilhil, que también significa semen (Farella, 1984: 73). 
dios Hablador y dios Llamador, ya que este hombre médico quiere reservarse esa información. Otras fuentes nos dan los nombres de los seres sobrenaturales que fueron creados para que vivieran en las diferentes montañas, pero los nombres varían según las versiones. Slim Curly nos dice:

El Chico que Regresa con una Turquesa y la Chica que Regresa con una Sola Mazorca de Maíz, se fueron a vivir al interior de Blanca Peak para es$\operatorname{tar}$ [vivir] en su interior.

El Chico que Regresa con una Mazorca de Maíz Amarilla y la Chica que Regresa con una Mazorca de Maíz Amarilla, se fueron a vivir a Mount Taylor...

El Chico de los Materiales y la Chica de las Joyas, quienes se fueron a vivir al interior de San Francisco Peaks...

El Chico del Maíz Blanco y la Chica del Maíz Amarillo se fueron al interior de Hesperus Mountain.

El Chico Polen y la Chica Saltamontes se fueron a vivir al interior de Huerfano Mountain.

El Chico del Rocío y la Chica del Rocío de las Hojas se fueron a vivir al interior de Gobernador Knob ${ }^{11}$ (Wyman, 1970: 211-212).

Aunque los nombres de los seres sobrenaturales que viven en el interior de las montañas varían en las diferentes versiones, todas ellas coinciden en que en el interior de esas montañas viven seres sobrenaturales que es lo que las convierte en sagradas. Las cuatro montañas tienen especial importancia para los Návajos, porque en esas montañas se quedaron a vivir de forma permanente el dios Llamador, el dios Hablador; Larga Vida y Felicidad que son los encargados de la ceremonia Hózhóóji, después de crear a los seres sobrenaturales que también habitarían en ellas (Wymar, 1970: 472). La Mujer Cambiante nos dice sobre esos cuatro seres sobrenaturales:

Aunque toda la Gente Sagrada tenía una apariencia maravillosa, cuatro en particular eran muy superiores al resto. El Dios Hablador de la cima de Blanca Peak, el Dios Llamador de la cima de San Francisco Peaks eran grandes hombres. Antes de conocerles, me sorprendió lo realmente maravillosos que esos hombres eran. Cada vez que los miraba yo pensaba:

${ }^{11}$ Estos fueron los doce personajes que de acuerdo con la versión de Slim Curly habían salido del hatillo que el Primer Hombre siempre llevaba consigo. 
¿de dónde vienen?... Y los que están sentados a la entrada, el que está sentado en la parte sur es llamado Larga Vida, y el otro sentado a la entrada en la parte norte es llamado Felicidad... (Wyman, 1970: 471-472).

Frank Mitchell (1978: 178) nos dice que esos seres sobrenaturales y otros que fueron creados en este momento se fueron a vivir y desaparecieron en el interior de las montañas, en las rocas y en los ríos. En esos lugares vivirían para siempre, sin nacimientos ni muerte. La gente que se fue a vivir en esos lugares sagrados vivirían eternamente. La presencia de esos seres sobrenaturales se siente en el silbido del viento y en los cánticos de los pájaros azules, en el crecimiento del maíz (Fishler, 1953: 92).

Esa Gente Sagrada que ayudó a los Návajos en el pasado continúan residiendo en lugares específicos entre las Cuatro Montañas Sagradas y están disponibles para ayudar a los que saben cómo y dónde comunicarse con ellos (Bitsuie, 1995: 3).

Para los Návajos, toda la tierra inserta entre sus cuatro montañas es sagrada, ya que fue creada especialmente para que ellos vivieran en ella exclusivamente (aunque también la tradición oral de otros grupos como Hopi, Paiutes, Havasupais y los Apaches de Oeste identifica ese lugar como su lugar de creación) y donde están sus orígenes.

En 1984, un hombre médico nos dice:

Todo lo que fue creado en la tierra donde nosotros vivimos hoy, entre las Cuatro Montañas Sagradas es sagrado (Parlow, 1988: 57).

En 1995, Roman Bitsuie escribió:

La tierra en la cual la gente de la Nación Návaja vive está definida y marcada por Cuatro Montañas Sagradas y cuatro ríos. La tierra entre esas fronteras es el lugar que los Návajos llaman «Din'e Be Kayah» que significa «Tierra Návaja». Esas montañas y todo lo que se encuentra entre ellas es sagrado. De acuerdo con las enseñanzas tradicionales, fue en esta tierra en la que el Creador quiso que los Návajos vivieran y todo lo que necesitaran sería proveído entre esas montañas... Esta tierra entre las Cuatro Montañas Sagradas es su Jerusalén, su Meca o su Belén (Bitsuie, 1995: 2). 
En en ese escenario entre las Cuatro Montañas Sagradas donde acontecieron todas las historias en las que están contenidas las creencias de los Návajos. Klara Bonsack y Harris Francis (1994: 2) nos dicen: «El paisaje es la unión física entre la gente del presente (los Návajos) que vive ahora y su pasado.» A lo que yo añadiría, que esa tierra donde acontecieron las historias, es la unión física y espiritual del Návajo no sólo en su pasado, sino con los seres sobrenaturales que son parte de la misma, porque como bien dice Bitsuie:

Esa Gente Sagrada que ayudó a los Návajos en el pasado continúan residiendo en lugares específicos entre las Cuatro Montañas Sagradas y están disponibles para ayudar a los que saben cómo y dónde comunicarse con ellos (Bitsuie, 1995: 3).

Dentro del espacio sagrado, delimitado por las cuatro montañas, hay lugares que tienen un significado especial para los Návajos, bien, porque allí nació un ser sobrenatural, o porque en ese lugar ocurrió un acontecimiento en el que este ser participó (Comunicación Personal, julio de 1995). En estos lugares, que siempre van asociados con historias, la presencia de los seres sobrenaturales es más evidente, y los Návajos visitan esos lugares para comunicarse con sus poderes.

Se puede decir que para los Návajos que no construyen edificios religiosos, el territorio inserto entre esas Cuatro Montañas Sagradas, es como una «gran catedral». Y esos lugares específicos, que aparecen en las historias orales son como pequeños altares o capillas dentro de esa catedral. Al igual que en una gran catedral, en la que hay capillas que tienen una especial importancia para ciertas personas, lo mismo ocurre con el territorio inserto entre las Cuatro Montañas Sagradas. Hay lugares específicos que son más importantes que otros para ciertas personas, sin que esto signifique que el resto del territorio no sea sagrado para ellas.

En el interior de esa "gran catedral», y en el interior del hogan, que es una réplica a pequeña escala de esa «gran catedral», es el lugar donde el Návajo se comunica con los seres sobrenaturales para mantener la armonía-felicidad, el objetivo de su presencia en este mundo.

Antes de desaparecer de forma tangible, esos seres sobrenaturales dejaron a los Návajos unas normas de comportamiento, para mantener o restaurar la armonía-felicidad consigo mismo y con el mundo que les rodea, que es el objetivo del Návajo en este mundo. Los medios de co- 
municación que el Návajo utiliza para comunicarse con los seres sobrenaturales son los siguientes: ofrecimientos, oraciones y los dibujos de arena. Que éstos iban a ser la forma de comunicación entre los seres sobrenaturales y los Návajos lo encontramos en la ceremonia Hózhóóji:

De ahora en adelante... vosotros (los Návajos) vais a ser los habitantes de la tierra, nosotros vamos a desaparecer de esta tierra y viviremos en el interior de las rocas, de las montañas, de las colinas, del agua. Cada vez que necesitéis nuestra ayuda por la razón que sea, id a esos lugares y depositad ofrendas y nosotros las aceptaremos (Mitchel F., 1978: 210).

El equilibrio entre las personas, la Gente de la Superficie de la Tierra y la Gente Sagrada es mantenido con los ofrecimientos... Los ofecimientos acompañados de canciones y oraciones, conectan cada familia con la Gente Sagrada que viven en el gran Hogan (se refiere al espacio inmerso entre las Cuatro Montañas Sagradas)... Los ofrecimientos son la forma que tenemos para comunicarnos con la Gente Sagrada (Parlow, 1988: 57).

Roberta Blackgoat nos dice sobre los ofrecimientos que el Návajo hace a los seres sobrenaturales:

En la forma de Vida Návajo (The Návajo Way), los ofrecimientos permiten al Návajo hablar con la gente espiritual y a los espíritus responder a los Návajos (Parlow, 1988: 51).

Pero como nos dicen los Návajos, esa comunicación sólo se puede llevar a cabo en el espacio delimitado por las Cuatro Montañas Sagradas, y en lugares específicos, donde ellos conocen, porque lo han aprendido de sus antepasados, a los que les fue comunicado por los seres sobrenaturales antes de desaparecer del mundo tangible (Parlow, 1988: 26).

A diferencia de otras religiones, los seres sobrenaturales de los Návajos no se pueden trasladar de lugar y lo mismo ocurre con sus ceremonias. John Kee Williams, un hombre médico, dice:

Sin la tierra, la religión Návaja es una ceremonia vacía... La Forma de Vida Návaja no se puede practicar fuera del mundo návajo y las ceremonias no tienen significado fuera de la tierra donde se originaron... Las ce- 
remonias nos recuerdan cómo vivir La Forma Návaja. Si no vivimos nuestra vida de acuerdo con lo que se nos dice en nuestras oraciones y canciones, entonces esas oraciones y canciones pierden su poder ${ }^{12}$ (Parlow, 1988: 104).

La explotación del uranio y del carbón: su impacto en el medio ambiente y en la salud de los návajos

Para los Návajos tradicionales, esa explotación significa más que la contaminación procedente de las minas de uranio y carbón. La extracción de los recursos naturales del interior de la Madre Tierra lleva consigo la destrucción y el abandono del mundo que les regalaron los seres sobrenaturales; la destrucción y el abandono de los lugares donde viven sus seres sobrenaturales y donde se comunican con ellos; la destrucción de su forma de vida que viene validada por sus creencias, y cuyo objetivo es el mantenimiento de la armonía con el mundo que les rodea.

$\mathrm{El}$ «libro sagrado» de los Návajos se encuentra escrito en la tierra, entre sus Cuatro Montañas Sagradas, siendo la tierra visible el soporte de las historias que allí tuvieron lugar y que conforman el sistema de creencias del Návajo. La destrucción de ese libro-tierra, no sólo es la destrucción física de la tierra, sino de las creencias que allí se encuentran.

Como nos dice un hombre médico que se opone a la explotación de los recursos energéticos: «El problema que estamos enfrentando ahora mismo (la explotación de los recursos minerales en el interior de las Cuatro Montañas Sagradas) es el último lloro de la Madre Tierra» (Parlow, 1988: 19).

La explotación de los recursos energéticos en la reserva návaja comenzó en los años veinte y todavía sigue teniendo lugar. Desde 1948 se han abierto más de 1.200 minas de uranio en la reserva localizadas principalmente en Lukachukai Mountains, Carrizo Mountains, Cameron, Globe, Tuba, Chilchibinto, en Arizona; White Canyon, Monumental Va-

\footnotetext{
${ }^{12}$ Forbes (Comunicación Personal, enero de 1996) dice que uno de los problemas que siempre se planteaba entre un grupo de Návajos que viven en Oakland, California, es que cuando se ponían enfermos no sabían si las ceremonias serían efectivas, ya que están fuera del lugar que les fue dado por los dioses, es decir de las Cuatro Montañas Sagradas.
} 


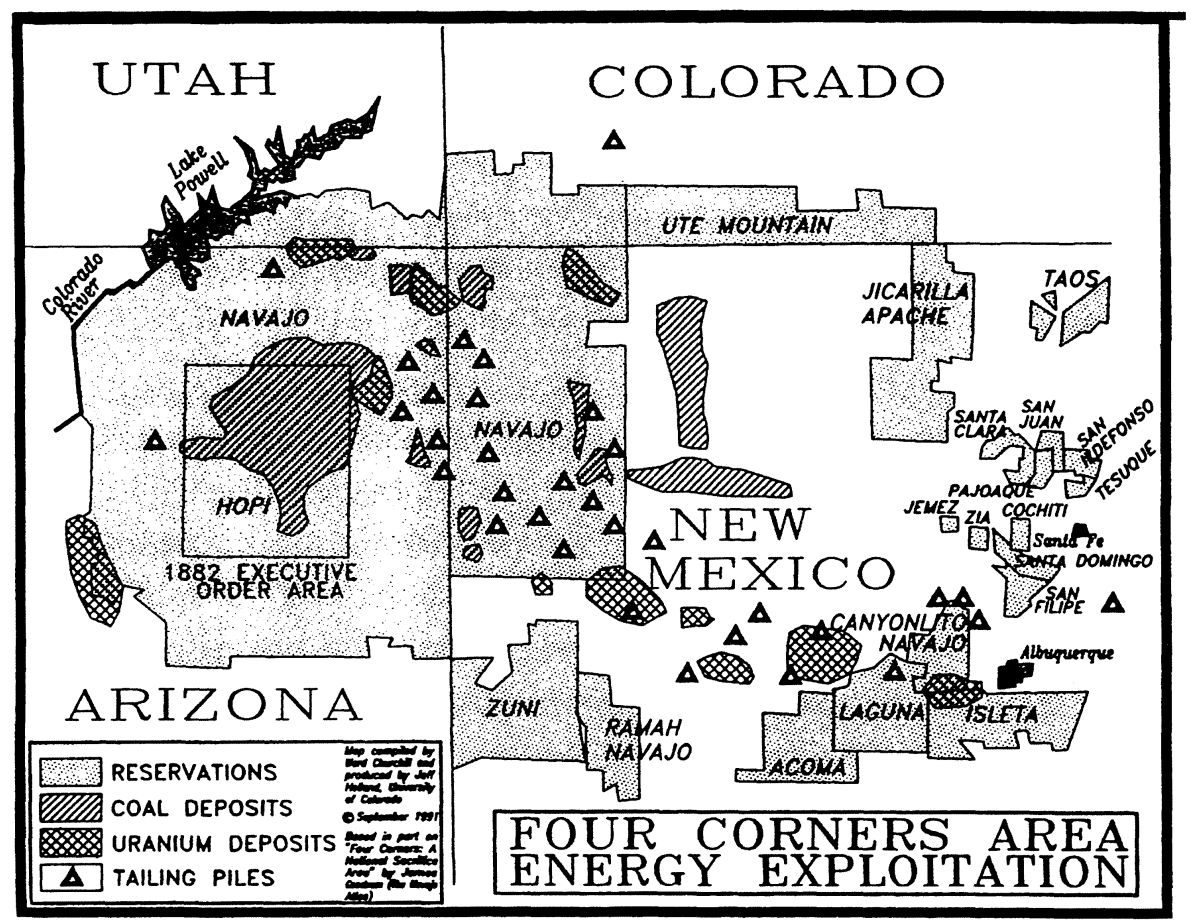

Fig. 2.

lley, Mexican Hat, en Utah; y Chuska Mountains, Shiprock, Church Rock, Bluewater, Grants, en Nuevo México (Eichstaedt, 1994: 36, 127). La mayoría de las minas de uranio que fueron abiertas en las áreas de Shiprock, Monument Valley y en el área de Tuba City, fueron abandonadas a finales de los setenta o principios de los ochenta ${ }^{13}$. Pero por la forma en que ese abandono se produjo, el peligro del uranio continúa hasta nuestros días, ya que las compañías responsables de la extracción del uranio dejaron abandonadas, en 24 lugares de la reserva, millones de toneladas de material radiactivo (Eichstaedt, 1994: 128). Estos vertidos de deshechos radiactivos están formados por las rocas donde se encontraba el uranio. Se calcula que estos deshechos todavía contienen

${ }^{13}$ Actualmente las minas de uranio en la reserva están localizadas principalmente en tres puntos en Nuevo México, Church Rock y sus alrededores, Sanostee y el área entre Shiprock y Two Grey Hills. 
$85 \%$ de la radiactividad original del uranio que permanece en un $99,9 \%$ de su volumen original (Folk-Williams, 1979: 15; Solomon y Wasserman, 1982: 178; Churchill y LaDuke, 1992: 248, 1996: 157; Matthiessen, 1986: 296).

Un estudio llevado a cabo en 1974, por el doctor Joseph Wagoner, investigador del Servicio de Salud Pública (Public Health Service), con 3.415 mineros que habían trabajado en las minas de uranio en la Meseta del Colorado, informa que 144 habían muerto de cáncer de pulmón. De acuerdo con sus estimaciones, este número alcanzaría los 200 en muy poco tiempo. Este estudio dice que solamente hubieran ocurrido 30 muertos de cáncer de pulmón en grupos similares que no hubieran sido expuestos a radiaciones provenientes del uranio (Eichstaedt, 1994: 106).

Otro estudio llevado a cabo en 1981, por Sarah Harvey, directora del programa Community Health Representative, encontró el doble de abortos espontáneos, y de niños nacidos muertos o con malformaciones congénitas en las familias que habían trabajado en las minas de uranio, que entre las familias que no estaban relacionadas con las mismas (Solomon y Wasserman, 1982: 198). En enero de 1981, la Doctora Evelyn Odin, pediatra que trabaja en Shiprock, dijo al periódico The Albuquerque Tribune que estaba impresionada por el gran número de niños que nacían prematuramente con microcefalias y otras malformaciones congénitas. Esta doctora da algunos ejemplos de niños que nacieron con el esófago y la tráquea unidos (Solomon y Wasserman, 1982: 186-187).

Un estudio llevado a cabo, desde 1969 a 1980, en Arizona, demostró que en el Condado de Coconino, los casos de malformaciones congénitas en los recién nacidos, eran dos veces superior a la media de los Estados Unidos. El mismo estudio dice que en el área de Cameron los niños nacidos con deformaciones congénitas son cinco veces superiores a la media nacional de ese país (Akwesasne Notes, 1989: 22).

Un estudio realizado en 1975, por la Agencia de Protección del Medio Ambiente de los Estados Unidos (Environmental Protection Agency), en el área de Cane Valley, Arizona, una de las zonas más ricas en minas de uranio en la reserva, y donde había un vertedero de materiales radiactivos que ocupaba una superficie de 8 hectáreas y una altura de 16,5 metros de alto, reveló que de 37 casas construidas allí, 16 habían utilizado materiales radiactivos procedentes de ese vertedero. Esos materiales, 
nos dice el informe, no sólo fueron utilizados para hacer los cimientos, como ha ocurrido en la zona de Gran Juction en el sur de Colorado, sino también para los suelos, las paredes interiores y exteriores, e incluso en las chimeneas (Barry, 1979: 25; Eichstaedt, 1994: 142-143). A pesar de que este estudio encontró niveles de radiactividad hasta tres veces superiores a los permitidos por el Departamento de Energía, no se tomó ninguna acción. Se dijo que los altos niveles de radiactividad medidos en las casas procedían de un vertedero radiactivo que se encuentra próximo a ellas (Eichstaedt, 1994: 142).

Otro de los grandes problemas de estas minas, que fueron clausuradas en los años setenta y ochenta, es que muchas veces son utilizadas como corrales, temporales y permanentes para el ganado. Las vacas y ovejas se contaminan como consecuencia de la existencia de radón, de beber el agua que mana de las minas o en los charcos, y comer el forraje contaminado con uranio y otros elementos tóxicos. Esta contaminación afecta a la carne de las ovejas y de las vacas, así como a su leche, que es consumida por los rancheros locales (Eichstaedt, 1994: 132-133).

Además, se da la circunstancia de que estas minas abandonadas son usadas regularmente por los Návajos como refugio cuando están atendiendo sus ganados durante las tormentas y el mal tiempo (Eichstaedt, 1994: 133).

En 1978, el Congreso de los Estados Unidos autorizó la limpieza de los restos de uranio procedente de las minas que habían sido dejados abandonados a lo largo de toda la reserva. Pero no fue hasta 1990, doce años más tarde, cuando el Departamento de Energía comenzó a vallar los vertederos y a colocar signos de peligro de radiactividad en esos lugares (Eichstaedt, 1994: 140-141) ${ }^{14}$. En 1983 los más de 1.000 lugares donde hay vertederos radiactivos en la reserva, fueron designados por la Agencia de Protección del Medio Ambiente de los Estados Unidos (Environmental Protection Agency) como «demasiado remotos» para garantizar los gastos para intentar su rehabilitación (Churchill, 1993: 271).

Perry Charley, un Návajo que trabaja en la oficina de Reclamación de

${ }^{14}$ En 1978 el Congreso de los Estados Unidos aprobó una ley Uranium Mill Takling Radiation Control Act, que ordenaba la limpieza de todos los lugares de procesamiento de uranio debido a los problemas ponteciales causados para la salud y el medio ambiente por la contaminación radiactiva (Eichstaedt, 1994: 128). 
las Minas Abandonadas ${ }^{15}$ (Návajo Abandoned Mine Land Reclamation), en Shiprock, nos dice que uno de los problemas con que se encuentra su oficina es cómo educar a los Návajos sobre el peligro de las radiaciones:

¿Cómo explicar a los Návajos sobre las partículas alfa o beta, sobre la radiación gama o el gas radón? No hay una palabra návaja para eso... Yo lo llamo vapor, pero ellos lo asocian con los baños de vapor ceremoniales, el cual es bueno (Eichtstaedt, 1994: 133).

\section{La central térmica de Four Corners}

Otra fuente importante de contaminación en la reserva es la central términca de Four Cornes, la cual entró en funcionamiento en 1963, y es una de las más grandes del mundo, localizada cerca de Shiprock, Nuevo México ${ }^{16}$. Esta central, que fue construida sin ningún sistema para el control de contaminación de aire, se ha calculado que emite diariamente 240 toneladas de hollín y 455 toneladas de gases contaminantes, óxidos de nitrato $\left(\mathrm{NO}_{\mathrm{X}}\right)$ y dióxidos de sulfuro $\left(\mathrm{SO}_{2}\right)$ (Black Mesa Report, 1973: 16, 19; Gordon, 1973: 48-50).

En su libro titulado Energy: A Crisis in Power, los doctores Holdren y Herrera, del Instituto de Tecnología de California, explican los «poderes» del dióxido de azufre:

Los dióxidos de azufre están considerados como los más peligrosos de todos los contaminantes del aire ${ }^{17}$. Están implicados en el aumento de muertes por bronquitis, enfisema pulmonar, cáncer de pulmón y otras enfermedades respiratorias. Se cree que concentraciones de dióxido de azufre tan pequeñas como 0,04 partes por millón (ppm) son adversas para la salud (Holdren y Herrera, 1971: 39).

15 Esta agencia está encargada de localizar las minas abandonadas y precintarlas para que no puedan volver a ser utilizadas de ninguna forma por los habitantes que residen cerca de las mismas.

16 Four Corners es una de las seis centrales construidas por un consorcio de 23 compañías semi-privadas conocido con las iniciales WEST (Estern Supply and Transmission Associates). Estas seis centrales suplen energía eléctrica al Sur de California, a las áreas de Tucson-Phoenix, al centro de Arizona, Las Vegas y algunas áreas de Nuevo México, Utah y Colorado (Gordon, 1973: 12).

17 Los dióxidos de azufre incluyen los dióxidos de azufre $\left(\mathrm{SO}^{2}\right)$ que constituyen la mayor parte de las emisiones, y los trióxidos de azufre $\left(\mathrm{SO}^{3}\right)$ que se forman por la oxidación del $\mathrm{SO}^{2}$ (Holdren y Herrera, 1971: 39, nota a pie de página). 
Según un estudio realizado por la Agencia Nacional Control de la Polución del Aire (National Air Pullution Control Agency), las concentraciones de dióxido de azufre en el Suroeste de los Estados Unidos son de dos partes por millón, es decir, cincuenta veces superiores a las cifras dadas por Holdren y Herrera como potencialmente peligrosas para la salud (Gordon, 1973: 50).

Otro de los problemas del dióxido de azufre es que, cuando se mezcla con el agua, se convierte en ácido sulfúrico, que produce la lluvia ácida que es altamente peligrosa para la vida de las plantas y de los animales.

\section{La explotación del carbón en Black Mesa (Arizona)}

Aunque fue en 1911 cuando los geólogos descubrieron en Black Mesa lo que consideraron era uno de los depósitos más extensos de carbón en el mundo, las Guerras Mundiales y la consiguiente falta de equipo retrasaron su extracción hasta los años sesenta (Gordon, 1973: 12).

En 1966, la compañía de carbón Peabody Coal, comenzó una serie de negociaciones con los Consejos Tribales de los Hopis y de los Návajos que concluyeron con la concesión, durante treinta y cinco años, de $259 \mathrm{~km}^{2}$ en la esquina noroeste de Black Mesa. De esos $259 \mathrm{~km}^{2}, 97$ estaban localizados en la reserva de los Návajos y 162 en un área compartida por Hopis y Návajos, conocida como el Área de Uso Conjunto (Blak Mesa Report, 1973: 5; Kammer, 1980: 78).

En 1970 y 1973, la Peabody Coal Company comenzó la explotación de carbón en Black Mesa en dos lugares diferentes, conocidos como la Mina de Black Mesa y la Mina de Kayenta (Dineh Alliance ${ }^{18}$, 1996: 2, 3 de marzo de 1996).

La proximidad del carbón a la superficie de la tierra en Black Mesa, permite que éste pueda ser extraído a cielo abierto, con el sistema de franjas, en lugar de minas subterráneas. Mediante ese sistema lo que se hace es romper y remover las capas superiores hasta llegar a las rocas

${ }_{18}$ Dineh Alliance es una organización formada de residentes de las comunidades de Black Mesa afectados por la explotación de las minas que se encuentran en ese área. Esta organización está basada en los valores tradicionales de los Návajos de mantener la armonía de la tierra. 
que cubren las vetas de carbón, lo que implica la destrucción de toda la vegetación.

Las compañías responsables de la extracción del carbón de Black Mesa dijeron que una vez que el carbón fuera extraído, las rocas se volverían a depositar en el lugar que se encontraban y así se restauraría la vegetación (Gordon, 1973: 78). En 1973, en un informe publicado sobre la explotación de carbón de Black Mesa, dice que eso no implica que la vegetación se pueda restaurar, ya que «las capas de rocas, antes fuertes y uniformes, se encuentran fragmentadas. El suelo productivo se mezcla con rocas infértiles, o incluso muchas veces el suelo fértil termina en el fondo y es cubierto por estas rocas infértiles» (Black Mesa Report, 1973: 10).

Otro de los problemas que hacen difícil ese proceso de reconstrucción de la vegetación, es que en el interior de la tierra, aparte del carbón, también hay filones de otros minerales, como hierro, manganeso y sulfuros. Cuando los sulfuros se mezclan con el agua de la lluvia o con la nieve, se convierten en ácido sulfúrico, que inhibe el crecimiento de las plantas. Este ácido sulfúrico es transportado por el agua a los campos de cultivo de los Návajos, que se encuentran cerca de las minas de carbón (Gordon, 1973: 64).

Una vez que el carbón ha sido extraído, se reduce a pequeños fragmentos y se transporta a las centrales de Mojave en Bullhead City, Nevada, situada a unos 410 kilómetros de Black Mesa, y a la planta Návajo in Page, Arizona, situada a 127 kilómetros. El carbón se lleva a la central de Mojave a través de unas tuberías subterráneas de 45 centímetros de diámetro ${ }^{19}$ (Black Mesa Report, 1973: 7; Benedek, 1993: 141; Bonsack y Harris, 1994: 150). Pero como nos dice Suzane Gordon:

La minería en Black Mesa y la construcción de centrales en el Suroeste de los Estados Unidos no sólo extraen el carbón de la tierra y contaminan el aire. Arrancarán al desierto una de sus comodidades más preciosa, el

${ }^{19}$ Según los residentes de Black Mesa, esas tuberías están siendo operadas sin los permisos requeridos; ya en 1985 la Peabody Coal pidió a la oficina de Minería de Superficie del gobierno de los Estados Unidos (US Goverment's Office of Surface Mining) el permiso para seguir explotando las minas cercanas a Black Mesa y a la Kayenta y no se lo dieron (Akwesasne Notes, 1990: 8); en 1995, la portavoz de Peabody Coal Company, Irene Crawford, dijo en 1995, que el permiso para la mina de Black Mesa está pendiente de ser concedido por el Departamento del Interior de los Estados Unidos (Norrell, 1995: 2). 
agua. El carbón (de Black Mesa) se transporta a Mojave a través de esas tuberías subterráneas, donde el carbón convertido casi en polvo, se mezcla con agua. El costo al medio ambiente y a la gente del Suroeste es de 10.800 litros de agua por minuto que se extrae de los acuíferos (Gordon, 1973: 12).

Cuando se firmaron los contratos de arrendamiento, la Peabody Coal Company excavó cinco pozos para extraer agua situados a unos 3 kilómetros de distancia uno de otro, y de acuerdo con esta compañía, esto no supondría ningún peligro para el abastecimiento de agua de la superficie (Black Mesa Report, 1973: 7-8). Sin embargo, hoy se sabe, que la explotación del carbón, ha originado que todo el ecosistema de la vertiente Dinnebito, en Black Mesa, se esté secando. Se calcula que si se sigue usando agua de los acuíferos en las cantidades indicadas anteriormente, los recursos de agua de Black Mesa se secarán en un período de quince años (Silberman, 1995: 28).

La compañía Peabody Coal dijo que el agua que iba a ser utilizada para la extracción del carbón no era de buena calidad para el uso doméstico, para la agricultura o para la industria. De hecho, se sabe que el agua que está siendo utilizada por la compañía de carbón quizás sea la mejor agua de toda la que se encuentra en la reserva (Black Mesa Report, 1973: 8).

En 1989, la compañía Peabody Coal planeaba seguir explotando otros $218 \mathrm{~km}^{2}$ de tierra al sur de Black Mesa, y, aunque la localización exacta se mantiene en secreto, se piensa que es Big Mountain, montaña de especial significado para los Návajos y también para los Hopis ${ }^{20}$ (Akwesasne Notes, 1990: 8). Este carbón se transportaría por tren a Long Beach, California, y posteriormente sería mandado por barco a Japón, que de esta forma espera terminar su dependencia en materias de carbón con Suráfrica (Parlow, 1989: 6).

El 11 de marzo de 1996, la organización Dineh Alliance, consiguió que la compañía Peabody Coal no consiguiera la renovación de los permisos necesarios para continuar con la explotación del carbón du-

${ }^{20}$ Según la información publicada en el periódico Akwesasne Notes (1990: 8), la prueba de que ese lugar es Big Mountain, es un mapa y papeles oficiales que fueron enviados por el Consejo Tribal Hopi al Juzgado Superior de Arizona, así como la solicitud de ese mismo Consejo del uso de más agua del Río Little Colorado y las razones para la misma. 
rante cinco años más en la Mina de Kayenta ${ }^{21}$. Aunque esta organización sabe, y espra, que Peabody apele esa decisión.

Actualmente los resultados de la expansión de la minería en Black Mesa son los siguientes:

Una alteración del paisaje, una importante reducción de los lugares donde las ovejas puedan pastar. Una invasión de plantas tóxicas, tolerantes al sol y venenosas para las ovejas, que han acabado con las plantas nativas y los pastos. Destrucción de los lugares sagrados para los Návajos... destrucción de plantas medicinales, destrucción de la vida salvaje, y una exarcebada contaminación del aire y de las aguas subterráneas (Silberman, 1995: 28).

En marzo de 1996, Dineh Alliance dijo sobre los efectos de la minería en Black Mesa:

Los acuíferos se están secando, causando la contaminación del agua que todavía existe y que está causando la muerte a las ovejas. El aire respirado por los residentes que viven cerca de las minas está contaminado con polvo procedente del carbón afectando a su salud y a la de sus animales... (Dineh Alliance, 1996: 3, 3 de marzo de 1996).

La explotación del carbón en Black Mesa fue responsable de la aprobación, el 22 de diciembre de 1974, de una ley conocida con el nombre Ley Pública 93-531 22. Esta ley obligaba a más de 11.000 Návajos y a unos 100 Hopis a abandonar Black Mesa, donde habían vivido desde tiempos inmemoriales, y a trasladarse a determinadas áreas dentro y fuera de la reserva ${ }^{23}$ (Parlow, 1988: 19).

${ }^{21}$ Esta sentencia fue dictada por un juez de Arizona, que dijo que la Peabody Coal no respetó lugares ceremoniales ni las tumbas considerados sagrados por los Návajos (Becenti, 1996: 1).

${ }_{22}$ Esta ley también es conocida con el nombre de Owen Bill (Akwesasne Notes, 1975: 32).

${ }_{23}$ El número de Návajos que tenían que abandonar su lugar de residencia ha variado de manera considerable a través de los años. En principio se dijo que iban a ser 3.500, luego 6.000 (Akwesasne Notes, 1979: 22). En diciembre de 1980, la Comisión de Realojamiento enumeró unos 2.801 hogares, con una población total de 9.525 (Lewis, Aktkinson y Masseto, 1981: 11-31); de acuerdo con Churchill (1992: 152), el número de Návajos forzados a realojarse es de 13.500. En un libro publicado en 1956, Churchill (1996: 121) habla de 17.500 personas afectadas por el realojo. Otras cifras publicadas el 30 de junio de 1986 en el periódico The Christian Science Monitor dicen que el número de Návajos que se tienen que realojar es de unos 15.000 (Sullivan, 1986: 7). 
De acuerdo con el Congreso de los Estados Unidos, la aprobación de la Ley Pública 93-531, conocida también como el Acta de Realojamiento (Relocation Act), supondría el final de cien años de disputa entre Hopis y Návajos (Parlow, 1988: 19). Para los Návajos y para los Hopis, que por esta ley estaban residiendo en el área equivocada, esto supuso el comienzo de un calvario que dura hasta nuestros días ${ }^{24}$.

En 1996, unas 250 familias návajas tradicionales que seguían viviendo en ese área de Black Mesa, siguen sufriendo problemas de salud importantes debido a los efectos del polvo del carbón (Yakama Nation Review, 1996: 1-2). La mayoría de sus ovejas, su principal y a veces único sustento, han muerto por beber agua contaminada (Dineh Alliance, 1996: 2). El 6 de abril de 1995, los residentes de Black Mesa presentaron un escrito al periódico Indian Country Today, donde decían: «Aunque no seamos científicos, cuando matamos nuestros animales, podemos ver manchas negras en sus órganos y tumores...» (Norrell, 1995) ${ }^{25}$.

¿Por qué se necesita ese carbón, es para el beneficio de los Návajos?, la respuesta es absolutamente no. Según el censo de los Estados Unidos de 1990 , el $54 \%$ de los Návajos utilizan madera para calentarse y el $75 \%$ carece de electricidad. Ese carbón es utilizado para alimentar las centrales térmicas que mandan electricidad a la ciudad de Los Ángeles, al departamento de Aguas y Energía de Los Ángeles California, Nevada Power Company y a la Oficina de Reclamación de los Estados Unidos, así como a las Compañías de Servicio Público de Arizona (Parlow, 1989: 6). La reserva de los Návajos sigue siendo un «País Tercermundista» dentro de uno de los países más ricos de todo el mundo.

${ }^{24}$ Todos los Návajos y los Hopis tradicionales coinciden en afirmar que esta disputa fue creada por el gobierno federal para beneficio de las compañías explotadoras de los recursos minerales. Esta fue la respuesta de algunos de mis informantes cuando yo les preguntaba sobre el reparto de tierras por la Ley Pública 93-531. Un tema muy interesante es como ven los Hopi tradicionales la explotación de los recursos minerales en Black Mesa y la Ley Pública 93-531.

${ }^{25}$ En abril de 1997 tuve la oportunidad de hablar con la hija de Katherine Ashki Smith, una de las ancianas que más se han opuesto al abandono de Black Mesa. Su familia no ha abandonado su hogan. Al preguntarle si eran molestado por el gobierno federal para abandonar su casa, me dijo que «ellos dejan notar su presencia». 


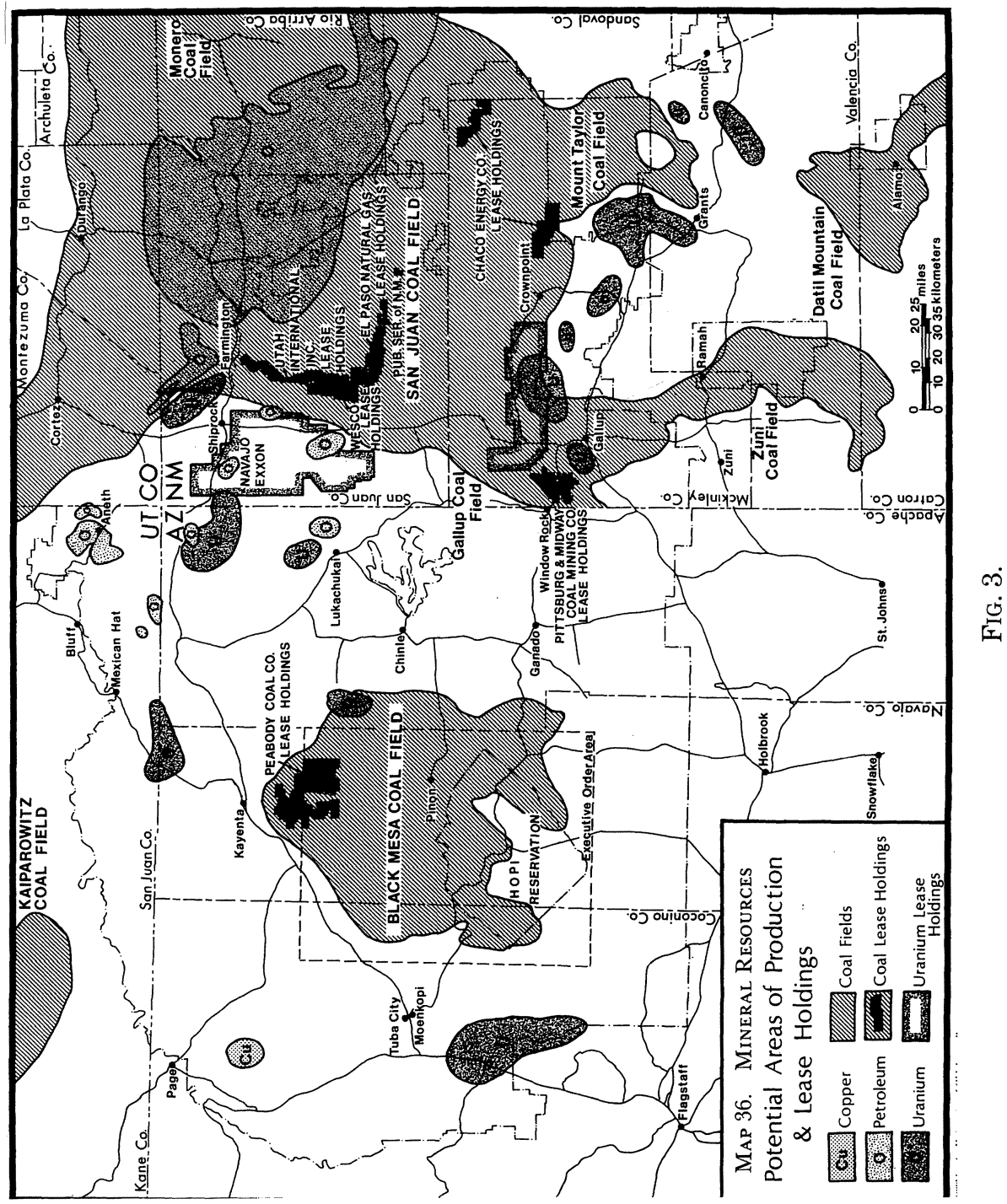

Conclusión

Cómo la explotación de los recursos energéticos en la reserva está afectando a los Návajos, se resume perfectamente en estas dos citas: 
Aunque es una lucha con diferentes armas, la estrategia del Departamento del Interior y de las compañías de carbón y otras compañías es como las viejas Guerras Indias. Hoy en día simplemente han cambiado las técnicas... La artillería y los soldados a caballo han sido reemplazados por las turbinas y generadores. El genocidio será menos directo. En lugar de matarlos (a los indios) con plomo (balas) en sus estómagos, los atacantes lo mandan contaminación al aire, sal a sus ríos, y les roban su preciosa agua de sus tierras. Los indios morirán porque morirá su Tierra... (Gordon, 1973: 14-15).

Esa tierra y las creencias que van con ella riegan y fertilizan la mente del Diné (Návajo), ayudándole a mantener su identidad en el «centro» del mundo (MacPerson, 1992: 3).

\section{BIBLIOGRAFÍA}

AKWESASNE NOTES

- (1975): Hopi and Navajo to Negotiate Land Use. 7 (2): 32-33.

- (1979): Relocation Threatens 6,000 Navajos. 11 (3): 22-23.

- (1989): Uranium in Arizona's Coconino County. A Fact Sheet. 20 (5): 22

1990 Peabody Coal Comes Closer to Big Mountain. 21 (6): 8.

Aronilth, Wilson Jr. S. F.: The Story of Earth \& Sky's Creation. Tsaile, Arizona: Navajo Community College.

Amsdem, Charles Avery (1932): «Navajo Origins», New Mexico Historical Review, 7 (3): 193-209.

BARRY, Tom (1979): «The Navajo Nation: Living and Breathing Radioactivity», Akwesasne Notes, otoño de 1979: 25-26.

Becenti, Deenise (1996): «Judge Says No To Mining Permit on Navajo Reservation», The Salt Lake Tribune, 27 de marzo de 1996, pp. 1-2.

BenedeK, Emily (1993): The Wind Wont't Know Me. A History of the Navajo-Hopi Land Dispute. New York: Vintage Books. A Division of Random House, Inc.

Bitsuie, Roman (1995): Holy Wind and Natural Law, 21 de abril de 1995.

BLACK MESA REPORT (1973): Environmental and Economic Issues: The Strip Mining on Black Mesa and the Coal-Burning Power Plants in the Southwest. Realizado por los profesores y alumnos del Instituto de Many Farms, Arizona. Santa Bárbara, California.

BonsACK, Klara, y Francis, Harris (1994): Navajo Sacred Places. Bloomington: Indiana University Press.

Churchill, Ward, y LADUKE, Winona (1992): «Native North America: The Political Economy of Radioactive Colonialism», en The State of Native America: Genocide, Colonization and Resistance. Editado por M. Annette Jaimes, Boston: South End Press. El mismo artículo en 1996 en Churchill, From a Native Son. Selected Essays on Indigenism, 1985-1995. South End Press, Boston, Massachusetts.

CHuchILL, Ward (1992): «The Earth is Our Mother. Struggles for American Indian Land and Liberation in the Contemporary United States», en The State of Native American: Genocide, Colonization and Resistance. En 1996 en Churchill, From a Native Son. Selected Essays on Indigenism, 1985-1995. 
- (1993): Struggle for the Land. Indigenours Resistance to Genocide, Ecocide and Expropiation in Contemporary North América. Common Courage Press. Monroe, Maine.

Dineh Alliance (1996): Legal Victory: Permit Repeal, 3 de marzo de 1996.

- (1996): Overview of the Diné (Návajo) Crisis, pp. 1-4.

Eichstaedt, Peter H. (1994): If You Poison Us. Uranium and Native Americans. Santa Fe, New Mexico: Red Crane Books.

Evers, Larry (1994): Between Sacred Mountains. Navajo Stories and Lessons from the Land. Tucson, Arizona: Sun Tracks and the University of Arizona Press.

FARELlA, John (1984): The Main Stalk. A Synthesis of Navajo Philosophy. Tucson, Arizona: The University of Arizona Press.

Fishler, Stanley A. (1953): «In the Beginning. A Navaho Creation Myth». University of Utah, Anthropological Papers, 13: 1-130.

FolK-Williams, John A. (1979): «Uranium Mining of Navajo Land», Akwesasne Notes, 11 (1): 15-18.

Goodman, James M. (1982): The Navajo Atlas. Environments, Resources, People and History of the Diné Bikeyah. University of Oklahoma Press: Norman.

Gordon, Suzanne (1973): Black Mesa. The Angel of Death. New York: The John Day Company.

HaILE, Berard OFM (1938): Origin Legend of the Navaho Enemyway, New Haven: Yale University Publications in Anthropology, n. ${ }^{\circ} 17$.

Holdren, John, y Herrera, Philip (1971): Energy: A Crisis in Power. Sierra Club. San Francisco and New York.

Kammer, Jerry (1980): The Second Long Walk: The Navajo-Hopi Land Dispute. Alburquerque: University of New Mexico Press.

LewIS, K. Roger; AKTKInson, Hawley y MAsseto, Sandra (1981): Report and Plan: Navajo and Hopi Indian Relocation Commission (NHIRC) Flagstaff, Arizona.

Matthiessen, Peter (1986): Indian Country, New York: The Viking Press.

MatThews, Washington (1994): Navaho Legends. Salt Lake City: University of Utah Press (publicado originalmente en 1897).

McPherson, Robert S. (1992): Sacred Land, Sacred View. Navajo Perceptions of the Four Corners Region. Provo: Brigham Young University, Charles Redd Center for Western Studies.

Mrtchell, Frank (1978): Navajo Blesingway Singer. The Autobiography of Frank Mitchell, 1881-1967. Editado por Charlotte Frisbie y David McAllester. Tucson, Arizona: University of Arizona Press.

NoRrell, Brenda (1995): «Coal Slurry Contaminates Spring. Peabody Western Coal Company Declared Unfit», en Indian Country Today, 6 de abril.

PARLow, Anita (1988): Cry Sacred Ground. Big Mountain USA. Washington D.C.: Christic Institüe.

- (1989): This Map is Not the Smoking Gun», Akwesasne Notes, 21 (2): 6.

SLEIGHT, Frederick W. (1951): «The Navajo Sacred Mountain of the East. A Controversy», El Palacio, 58 (12): 379-397.

Solomon, Norman, y WASSERMAN, Harvey (1982): «Uranium Milling and the Church Rock Disaster», en Killing Our Own. The Disaster of America's Experience With Atomic Radiation, New York: Delacorte Press.

Silberman, Tracy (1995): «Green Hope for Black Mesa», Earth Island Journal, Fall, 1995, pp. 28-29.

Sullivan, Cherry (1986): «Navajo Indians Resist US Law Forcing them to Yield Land to Hopis, The Christian Sicience Monitor, 30 de junio, pp. 1, 6-7.

Watson, Editha (1964): «Navajo Sacred Places. Window Rock, Arizona», Navajo Tribal Museum, Navajoland Publications. 
WithersPoon, Gary (1975): «The Central Concepts of Navajo World View (II)», Linguistic: An International Review, 161: 69-87.

- (1977): Language and Art in the Navajo Universe. Ann Arbor: University of Michigan Press.

- (1995): Dynamic Symmetry and Holistic Asymmetry in Navajo and Western Art and Cosmology, Peter Lang Publishing.

WiLkins, David (1987): Diné Bibeehz'aanii. A Handbook of the Navajo Government. Tsaile, Arizona: Navajo Community College Press.

Wyman, Leland C. (1957): Beautyway: A Navaho Ceremonial. Bollingen Series LIII, Pantheon Books.

(1970): Blessingway. Tucson: University of Arizona Press.

(1983): Southwest Indian Drypainting. School of American Resear. Santa Fe. University of New Mexico Press. Albuquerque.

Yakama Nation Review (1996): Report: Navajo Spirits Visiting Big Mountain. 7 de junio. 27 (3): $1-2$.

ABSTRACT: One of the biggest problems the Navajos are suffering nowadays is the exploitation of the natural resources on their reservation. That exploitation is taking more than the coal and uranium from the interior of the land, is destroying what the Navajos considered is their Fifth World. This article will analyze why the physical destruction of the land implies also the spiritual destruction of the Navajos. 